\title{
Microwave Modulation of a Quantum-Well Laser with and without External Optical Injection
}

\author{
X. Jin and S. L. Chuang, Fellow, IEEE
}

\begin{abstract}
We compare three microwave modulation methods experimentally and theoretically using a semiconductor quantum-well (QW) Fabry-Perot test laser: 1) direct microwave current modulation of the test laser (electrical modulation); 2) optical modulation by an external single-wavelength pump laser with a modulated optical injection power; and 3) electrical modulation of the test laser that is injection locked by an external single-wavelength pump laser with a constant injection power. This is the first direct comparison of the three modulation methods on the same QW laser, to the best of our knowledge. The bandwidth of optical absorption modulation is $7.7 \mathrm{GHz}$, which is 1.45 times the direct electrical modulation bandwidth $(5.3 \mathrm{GHz})$ at a bias current of $30 \mathrm{~mA}$ in the test laser. On the other hand, the electrical modulation of the test laser under injection-locking condition has a significantly higher modulation bandwidth (10.5 GHz) than both the electrical and optical modulation methods.
\end{abstract}

Index Terms-Modulation, optical injection, semiconductor laser.

\section{INTRODUCTION}

$\mathbf{H}$ IGH-BANDWIDTH, single-mode, and low-chirp lasers are highly desirable for long-haul and high-bit-rate optical communications. A particular advantage of semiconductor lasers is that amplitude modulation can be achieved simply by varying the laser bias current, which is called direct microwave modulation or electrical modulation. Electrical modulation is the simplest method to realize modulation in semiconductor lasers; however, the modulation response in this case suffers a severe low-frequency rolloff due to carrier transport and parasitic effects, which limits the laser performance [1], [2]. Optical modulation can improve the modulation response by avoiding electron-hole transport effects in separate confinement heterostructure $(\mathrm{SCH})$ quantum-well $(\mathrm{QW})$ lasers and circuit parasitic effects, thereby giving the intrinsic modulation response [3], [4]. The bandwidth improvement is usually around $20 \%-50 \%$. More recently, several research groups have demonstrated that the modulation bandwidth of an injection-locked semiconductor laser shows significant improvement in the modulation characteristics relative to electrical modulation [5], [6],[7].

In this letter, we report on the first direct comparison of electrical modulation, optical modulation, and electrical modulation under injection-locking condition using the same InGaAsP semiconductor Fabry-Perot (FP) QW laser. By performing optical modulation in the absorption region of

Manuscript received July 5, 2000; revised March 28, 2001.

The authors are with the Department of Electrical and Computer Engineering,

University of Illinois at Urbana-Champaign, Urbana, IL 61801 USA.

Publisher Item Identifier S 1041-1135(01)05584-7.

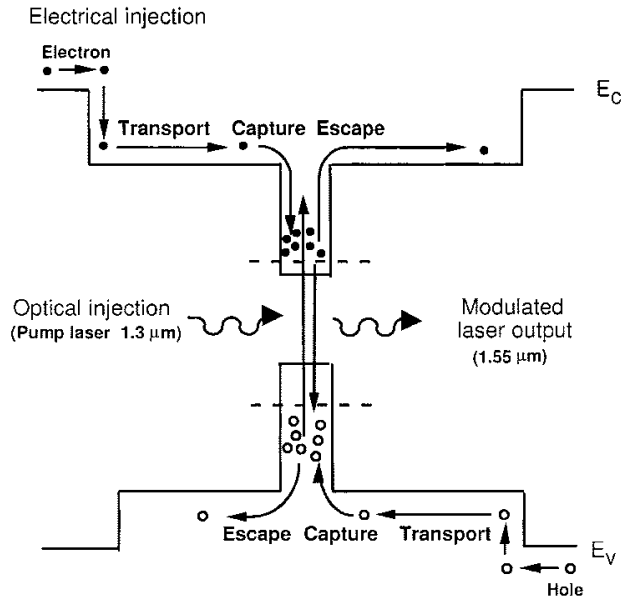

Fig. 1. QW energy band diagram for high-speed modulation by electrical or optical injection. For electrical injection, the carriers have to transport and get captured by the QWs. For optical injection, we choose the optical energy of the external pump laser in the absorption spectral region such that carriers are generated directly in the QWs.

semiconductor lasers, the modulation bandwidth increases by $45 \%$ compared to the electrical modulation bandwidth. By injection locking the direct microwave modulated test laser, we increase the modulation bandwidth further by $100 \%$. We also show the enhancement of the relaxation frequency of the test laser under injection-locking condition both experimentally and theoretically and realize a 3.5 times improvement in electrical modulation bandwidth.

\section{RESULTS AND DISCUSSION}

A schematic of high-speed modulation by electrical injection and optical injection is shown in Fig. 1. In electrical modulation, a small microwave modulation current is added to the dc current of a semiconductor laser, which produces a modulation in the laser output power. The electrical modulation response includes parasitic and transport effects. Parasitic effects arise from the bias circuit and the shunting of modulation current around the active layer. The injected carriers transport through the $\mathrm{SCH}$ region and are captured into the QWs before recombining by stimulated emission processes. In the SCH QW lasers, the time constants for the carrier transport, capture, and escape into and from QWs are usually defined to characterize the carrier transport processes [1] and give a parasitic-like rolloff in the modulation response, which is indistinguishable from parasitic effects. In QW lasers, the carrier transport effect is an important limit for multi-QW laser modulation bandwidth. The electrical modulation response of a compressively strained InGaAsP QW FP 


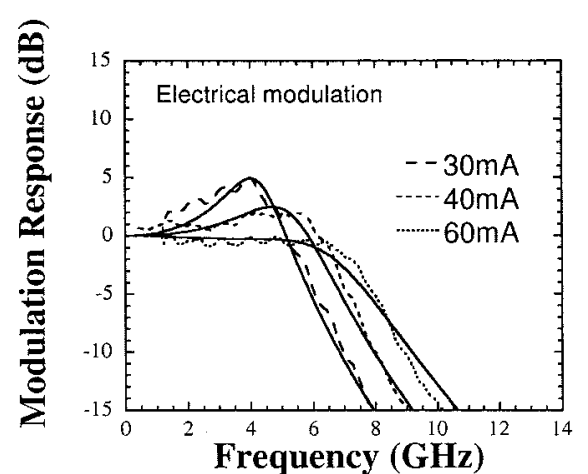

(a)

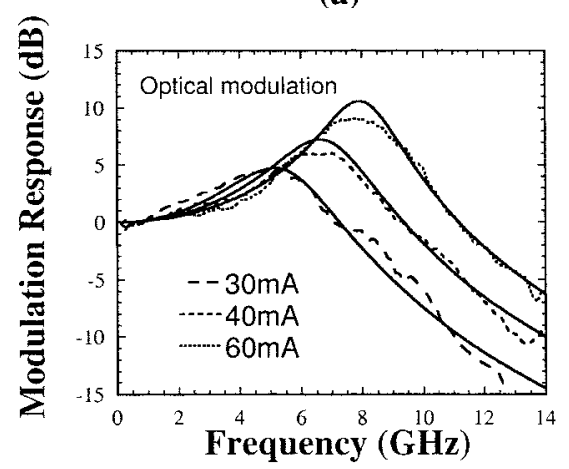

(b)

Fig. 2. (a) Electrical modulation responses at test laser bias of 30, 40, and 60 mA. (b) Optical absorption modulation responses at test laser bias of 30, 40, and $60 \mathrm{~mA}$. Dashed lines: Experimental data. Solid lines: Theoretical calculation.

laser is shown in Fig. 2(a). The dashed lines are experimental data, and the solid lines are theory. We realize a 4-GHz relaxation frequency and a 5.3- $\mathrm{GHz}$ bandwidth at current bias of 30 $\mathrm{mA}$. When the current bias increases, the relaxation peaks drop off very quickly. At $60-\mathrm{mA}$ bias, the relaxation peak is smoothed out by the additional rolloff frequency, which is $7 \mathrm{GHz}$, and modulation bandwidth will not improve for higher biases.

For optical modulation, a pump laser is electrically modulated to provide a modulated optical pump signal for the test laser, which is biased by a dc current above threshold. The modulated light is injected into the test laser cavity from the facet, and the pump wavelength is chosen to be in the absorption range of the test laser QWs. The pump light produces electron-hole pairs directly in the QWs. Therefore, carrier transport through the $\mathrm{SCH}$ region is not required for lasing action. In this way, optical modulation removes the low frequency rolloff due to the transport and parasitic effects and gives the intrinsic modulation response of the test laser. The modulation response of an optically modulated QW laser is shown in Fig. 2(b). The dashed lines are experimental data, and the solid lines are theory [4]. For a current bias of $30 \mathrm{~mA}$, we show that the bandwidth is increased to $7.7 \mathrm{GHz}$, which is $45 \%$ higher than the electrical modulation value. Compared to electrical modulation, the main difference between the two responses is the low-frequency rolloff. Without the limit of parasitic and transport effects, optical modulation has higher relaxation peaks that do not decrease at $60 \mathrm{~mA}$ bias. In general, the modulation response is improved by optical modulation. However, the optical modulation signal is usually $10-15 \mathrm{~dB}$ lower than that of electrical modulation due to high losses in the op-

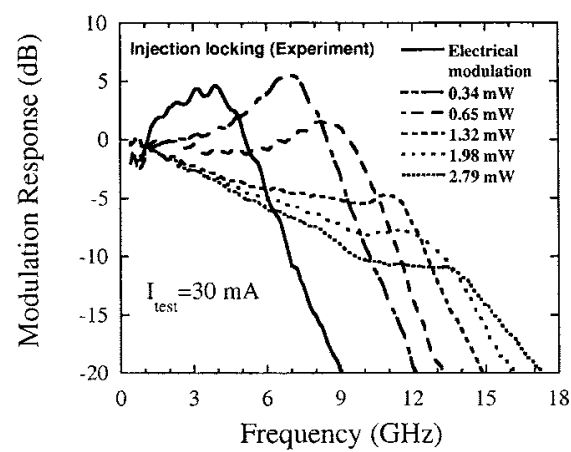

(a)

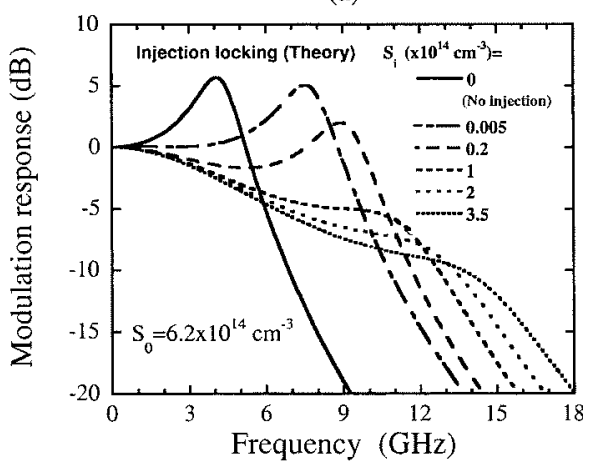

(b)

Fig. 3. (a) Experimental data of the electrical modulation response of the injection-locked test laser with a fixed test laser bias of $30 \mathrm{~mA}$ and injection powers of $0,0.34,0.65,1.32,1.98$, and $2.79 \mathrm{~mW}$. (b) Theoretical calculation of the electrical modulation response of the injection-locked test laser with a fixed test laser photon density $S_{o}=6.2 \times 10^{14} \mathrm{~cm}^{-3}$. The injected photon number $\left(S_{i}\right)$ varies from zero (no optical pump) to $3.5 \times 10^{14} \mathrm{~cm}^{-3}$ and is proportional to the injection power.

tical path such as the coupling between fiber and the pump laser. This means the conversion efficiency from electrical signal to optical signal is very low for optical modulation. For both cases, the fluctuation of the carrier density in the test laser cavity creates frequency chirping while modulating the test laser, which is also undesirable.

Electrical modulation of injection-locked semiconductor lasers is an attractive method to ensure sufficient signal conversion, single-mode operation, and eliminate mode partition noise as well as improve modulation bandwidth [5]. Injection locking requires two semiconductor lasers. When light from a single-wavelength master laser is injected into the test laser (or slave laser) oscillating above threshold, the injected radiation competes with the spontaneous emission of the laser being amplified. If the optical frequency of the injected light is close to the eigenfrequency of the unperturbed test laser, the test laser will adjust its frequency and coherence properties to those of the injected light. Once a perfect locking state is reached, all of the power in the test laser is emitted at the optical frequency of the master laser. This phenomenon is known as injection locking. The wavelength of a laser usually shifts for electrical modulation and optical modulation. However, the wavelength of an injection-locked laser is fixed by the external signal. In this way, low-chirp single-wavelength modulation can be achieved. In addition, the electrical-optical conversion efficiency of electrical modulation under injection-locking condition is the same as direct electrical modulation. 
TABLE I

COMPARISON OF THREE MODULATION EXPERIMENTS

\begin{tabular}{c|c|c|c}
\hline & $\begin{array}{c}\text { (i) Electrical } \\
\text { modulation }\end{array}$ & $\begin{array}{c}\text { (ii) Optical } \\
\text { modulation }\end{array}$ & $\begin{array}{c}\text { (iii) Electrical } \\
\text { modulation with } \\
\text { injection locking }\end{array}$ \\
\hline \hline Test laser & $\begin{array}{c}\text { dc bias and } \\
\text { ac current }\end{array}$ & dc bias & $\begin{array}{c}\text { dc bias and } \\
\text { ac current }\end{array}$ \\
\hline Pump laser & Not used & $\begin{array}{c}\text { - Single-wavelength } \\
\text { - Constant power } \\
\text { plus small-signal } \\
\text { modulation }\end{array}$ & $\begin{array}{c}\text { - Single-wavelength } \\
\text { near a resonant } \\
\text { mode of the test laser } \\
\text { - Constant power }\end{array}$ \\
\hline 3 dB bandwidth & $f_{3 d B}$ & $1.2 \sim 1.5$ times $f_{3 d B}$ & $2 \sim 3$ times $f_{3 d B}$ \\
\hline $\begin{array}{c}\text { Electrical-optical } \\
\text { conversion efficiency }\end{array}$ & Hight & Low & High \\
\hline Chirp & Hight & High & Low \\
\hline $\begin{array}{c}\text { Low frequency } \\
\text { roll-off }\end{array}$ & Existent & $\begin{array}{c}\text { Almost } \\
\text { absent }\end{array}$ & Existent \\
\hline
\end{tabular}

The main advantage for the electrical modulation of an injection-locked laser is the large increase in modulation bandwidth. The small-signal amplitude modulation response of the injection-locked test laser is measured when a dc master laser signal is injected into the electrically modulated test laser. We carefully checked the detuning $(-15 \mathrm{GHz})$ at each injection power to ensure that our experiment was within the range of the stable-locked operation. The minimum injected power level for injection locking at this condition is around $0.3 \mathrm{~mW}$. When injection locking takes place, the relaxation oscillation frequency shifts to high frequency. Fig. 3(a) shows the modulation response of the injection-locked test laser under a constant test laser bias of $30 \mathrm{~mA}$ at different injection powers $(0,0.34,0.65$, $1.32,1.98$, and $2.79 \mathrm{~mW})$. The power of the master laser is measured before it is injected into the test laser. The electrical modulation response of the test laser without injection (solid curve) is also shown in Fig. 3(a) for comparison. At 30-mA laser bias, the 3-dB bandwidth of the injection-locked laser at a pump power of $1.32 \mathrm{~mW}$ is $10.5 \mathrm{GHz}$, which is twice the electrical modulation bandwidth. The relaxation frequency increases with increasing injection power and reaches $14 \mathrm{GHz}$ at an injection power around $2.79 \mathrm{~mW}$, which is about 3.5 times the electrical modulation value. For an injection-locked FP laser, the total photon number in the test laser cavity increases very little under injection (by only a few percent at high-bias current). This means the improvement of relaxation frequency is not from the increase of the total optical power of the test laser. The main reason for the bandwidth enhancement is amplitude-phase coupling characteristics of the injection-locked laser system [5], [6], [8], [9]. In an injection-locked laser system, the injected photon term connects the amplitude and phase of the slave laser. The phase term must be counted to obtain amplitude value. Our research also shows theoretically and experimentally that this amplitude-phase coupling in the rate equations leads to the gain change (caused by nonlinear gain saturation terms) [5] and the linear relation between the injected photon and the relaxation resonance frequency squared [8]. Generally, any change in the injection power or the gain will alter the relaxation resonance frequency [8]. In addition, any change in the detuning of the master and free-running slave will also alter the relaxation resonance frequency. Note that this modulation method dose not remove the transport effect of QW structures and the parasitic effect of the bias circuit. As shown in Fig. 3, there is a drop-off of the modulation response at low frequencies. This phenomenon also appears in the experimental curves in [7]. Our theoretical calculation results are shown in Fig. 3(b). The minimum injected photon density for injection locking is around $1 \times 10^{11} \mathrm{~cm}^{-3}$. We assume that the photon density in the test laser is constant $\left(S_{\circ}=6.2 \times 10^{14} \mathrm{~cm}^{-3}\right)$. The injected photon number varies from zero (no optical pump) to $3.5 \times 10^{14} \mathrm{~cm}^{-3}$ and is proportional to the injection power. Actually, under strong injection, the circulating power within the laser cavity will not remain fixed at the free-running value. It can show an increase in the region of stable injection-locked operation, which will further increase the relaxation oscillation frequency. To accurately model the modulation response, we include an additional $7-\mathrm{GHz}$ roll-off frequency into the intrinsic response of injection-locked lasers. Our calculated responses are in good agreement with experimental data and show the enhancement of modulation bandwidth with injection locking. The comparison of three modulation methods is summarized in Table I.

In conclusion, we report a direct comparison between three modulation methods: 1) electrical modulation; 2) optical modulation; and 3) electrical modulation under injection locking using the same FP QW laser. At a test laser bias current of 30 $\mathrm{mA}$, the electrical modulation can realize a 5.3-GHz modulation bandwidth, and the optical absorption modulation can improve the bandwidth to $7.7 \mathrm{GHz}$, which is about a $45 \%$ improvement. The modulation bandwidth of an injection-locked laser can be $10.5 \mathrm{GHz}$, which is twice the electrical modulation bandwidth. We show that injection locking is a very efficient method to improve modulation performance of semiconductor lasers.

\section{REFERENCES}

[1] R. Nagarajan, T. Fukushima, S. W. Corzine, and J. E. Bowers, "Effects of carrier transport on high-speed quantum well lasers," Appl. Phys. Lett., vol. 59, pp. 1835-1837, 1991.

[2] C. Y. Tsai, F. P. Shih, T. L. Sung, T. Y. Wu, C. H. Chen, and C. Y. Tsai, "A small-signal analysis of the modulation response of high-speed quantum-well lasers: effects of spectral hole burning, carrier heating, and carrier diffusion-capture-escape," IEEE J. Quantum Electron., vol. 33, pp. 2084-2096, Nov. 1997.

[3] C. H. Lange and C. B. Su, "Theory and experiment of the parasitic-free frequency response measurement technique using facet-pumped optical modulation in semiconductor diode lasers," Appl. Phys. Lett., vol. 55, pp. 1704-1706, 1989.

[4] T. Keating, X. Jin, S. L. Chuang, and K. Hess, "Temperature dependence of electrical and optical modulation response of quantum-well lasers," IEEE J. Quantum Electron., vol. 35, pp. 1526-1534, Oct. 1999.

[5] T. B. Simpson and J. M. Liu, "Enhanced modulation bandwidth in injection-locked semiconductor lasers," IEEE Photon. Technol. Lett., vol. 9, pp. 1322-1324, Oct. 1997.

[6] J. Wang, M. K. Haldar, L. Lin, and F. V. C. Mendis, "Enhancement of modulation bandwidth of laser diodes by injection locking," IEEE Photon. Technol. Lett., vol. 8, pp. 34-36, Jan. 1996.

[7] X. J. Meng, T. Chau, and M. C. Wu, "Experimental demonstration of modulation bandwidth enhancement in distributed feedback lasers with external light injection," Electron. Lett., vol. 34, pp. 2031-2032, 1998.

[8] L. Lin, "Static and dynamic properties of injection-locked semiconductor lasers," IEEE J. Quantum Electron., vol. 30, pp. 1701-1708, Aug. 1994.

[9] G. Yabre, "Effect of relatively strong light injection on chirp-to-power ratio and $3 \mathrm{~dB}$ bandwidth of directly modulated semiconductor lasers," J. Lightwave Technol., vol. 14, pp. 2367-2373, Oct. 1996. 